\title{
Metastatic breast cancer cells in the bone marrow microenvironment: novel insights into oncoprotection
}

\author{
Shyam A. Patel, \\ Division of Hematology/Oncology, Department of Medicine, New Jersey Medical School, \\ University of Medicine and Dentistry of New Jersey, MSB, Room E-579, 185 South Orange \\ Avenue, Newark, NJ 07103, USA. Graduate School of Biomedical Sciences, University of \\ Medicine and Dentistry of New Jersey, Newark, NJ, USA
}

\section{Meneka A. Dave,}

Division of Hematology/Oncology, Department of Medicine, New Jersey Medical School, University of Medicine and Dentistry of New Jersey, MSB, Room E-579, 185 South Orange Avenue, Newark, NJ 07103, USA

\author{
Raghav G. Murthy, \\ Division of Hematology/Oncology, Department of Medicine, New Jersey Medical School, \\ University of Medicine and Dentistry of New Jersey, MSB, Room E-579, 185 South Orange \\ Avenue, Newark, NJ 07103, USA
}

Karim Y. Helmy, and

Division of Hematology/Oncology, Department of Medicine, New Jersey Medical School, University of Medicine and Dentistry of New Jersey, MSB, Room E-579, 185 South Orange Avenue, Newark, NJ 07103, USA. Graduate School of Biomedical Sciences, University of Medicine and Dentistry of New Jersey, Newark, NJ, USA

\section{Pranela Rameshwar}

Division of Hematology/Oncology, Department of Medicine, New Jersey Medical School, University of Medicine and Dentistry of New Jersey, MSB, Room E-579, 185 South Orange Avenue, Newark, NJ 07103, USA

Pranela Rameshwar: rameshwa@umdnj.edu

\section{Abstract}

Among all cancers, malignancies of the breast are the second leading cause of cancer death in the United States after carcinoma of the lung. One of the major factors considered when assessing the prognosis of breast cancer patients is whether the tumor has metastasized to distant organs. Although the exact phenotype of the malignant cells responsible for metastasis and dormancy is still unknown, growing evidence has revealed that they may have stem cell-like properties that may account for resistance to chemotherapy and radiation. One process that has been attributed to primary tumor metastasis is the epithelial-to-mesenchymal transition. In this review, we specifically discuss breast cancer dissemination to the bone marrow and factors that ultimately serve to shelter and promote tumor growth, including the complex relationship between mesenchymal stem cells (MSCs) and various aspects of the immune system, carcinoma-associated fibroblasts, and the diverse components of the tumor microenvironment. A better understanding of

(C) Springer-Verlag 2010

Correspondence to: Pranela Rameshwar, rameshwa@umdn j. edu.

This work was done at UMDNJ-New Jersey Medical School, Department of Medicine, Newark, NJ 07103.

Conflict of interest None. 
the journey from the primary tumor site to the bone marrow and subsequently the oncoprotective role of MSCs and other factors within that microenvironment can potentially lead to development of novel therapeutic targets.

\section{Keywords}

Oncoprotection; Cancer stem cell; Microenvironment; Metastasis

\section{Overview}

Breast cancer currently ranks as the second leading cause of cancer death and the most common cancer affecting women in the United States, with an annual incidence of approximately 175,000 and death rate of greater than 40,000 per year [1]. The histological origins of breast cancer span from the ductal epithelia, myoepithelia, and lobular elements [2]. Classification of cancers has always posed a major challenge to scientists. The major categories currently include luminal A, luminal B, basal-like, and human epidermal growth factor receptor 2 (HER2)-positive [3-6] (Table 1). Recently, the use of gene expression microarrays has shed insight into the classification of various breast cancer subtypes, and these studies have demonstrated important overlap among subtypes since tumors demonstrate a high degree of molecular and genetic heterogeneity [3].

Although there are a variety of known subtypes for breast cancer, particular attention in the arena of breast tumor stem cell biology has focused on the luminal versus basal-like cancers because each has been proposed to be the origin of stem-like tumor cells that allow for cancer maintenance. Basal-like cancers have been shown to carry a worse prognosis than luminal subgroups and are found in the African American population at a higher frequency than in other racial groups [7]. These cancers are generally high grade, suggesting that they may harbor stem-like features that allow for tumor survival and/or self-renewal [4]. Basallike cancers also demonstrate some overlap with triple-negative cancers [4]. Different subtypes can be distinguished molecularly by gene expression signatures, such as expression of cytokeratins 5, 6, and 14 by basal-like cancers, and expression of cytokeratins 8 and 18 by luminal cancers [5, 8]. Other minor classifications, such as the claudin ${ }^{\text {lo }}$ and claudin ${ }^{\text {hi }}$ subgroups, have been identified, but their clinical significance remains to be determined [9].

Although these classifications have been established for many years, the categorization appears to be more complex. Recently, a signal transduction pathway-based scheme for classifying breast cancer has been developed [3]. Each subtype demonstrates specific alterations at the genomic level. The advantage to this classification scheme is that pharmacological therapy against certain pathways, such as Akt signaling, can be tailored toward specific patients.

Molecular subtyping of breast cancer is underway and may be more challenging than anticipated based on the high level of heterogeneity among cancer cells [4]. This heterogeneity may be due to the high numbers of somatic mutational events during cancer progression [3]. In order to develop novel therapeutic strategies, the heterogeneity must be understood, and cellular subtypes must be characterized [3]. Each element of heterogeneity may represent a mechanism of oncogenesis that can be targeted by pharmacological therapy [3]. With the discovery that cancers are more heterogeneous than once believed, personalized therapy for breast cancer may finds its way into the clinic. However, methods for genomic analyses must first become optimized [3]. 


\section{Epithelial-to-mesenchymal transition: explanations for tumor dissemination}

One of the most important predictors of survival in patients with breast cancer is the presence or absence of distant metastasis [10]. The process of metastasis has long been known to encompass diverse biological events at the cellular and molecular levels. A major theory on the origins of metastatic cells arises from principles of the epithelial-tomesenchymal transition (EMT), an event that normally occurs during gastrulation, wound healing and repair, tissue development, tissue fibrosis, and renal tubule degeneration in chronic kidney disease $[11,12]$. EMT in cancer refers to a dynamic cellular process during which cells lose cellular polarity and attain a mesenchymal-like or fibroblastoid phenotype at the primary site of tumor [11]. Among the molecular changes are loss of E-cadherin, occludin, and $\beta 4$-integrin with gain of vimentin, fibronectin, $\mathrm{N}$-cadherin, and $\alpha$-smooth muscle actin [13, 14] (Fig. 1).

The phenotypic changes in EMT are frequently accompanied by functional alterations in cellular behavior. These changes may be relevant to functions to confer an increase in invasiveness with enhanced motility. For example, the epithelial tissue consists of monolayer of cells that tightly interact with each other to form an architect that protects against tissue damage [15]. E-cadherins, desmosomes, claudins, and occludins allow the epithelial cells to have strong attachments to their basement membranes and to each other. Downregulation of E-cadherin has been associated with a poor prognosis in cancer [14]. Upon EMT, actin stress fibers become remodeled in a manner that facilitates cellular motility [15]. The gain of vimentin, an intermediate filament protein that strengthens cellular integrity and promotes cell survival and migration, may facilitate the EMT process [16]. Mesenchymal-like cells are thought to have properties that allow for separation from the underlying connective tissue, and weak intercellular contact by the mesenchymal-like tumor cells facilitates migrational events $[12,14]$. Thus, the epithelial plasticity associated with carcinoma progression may underlie metastatic tendencies [12].

Among the major factors that mediate these events are Snail, Twist, Slug, Six 1, zinc finger E-box protein-1 (ZEB1), and Cripto, most of which have suppressive effects on E-cadherin expression [14]. ZEB1 functions in anchorage-independent growth in non-small cell lung carcinoma [13]. TGF- $\beta$ is also a major player in EMT, and this cytokine is particularly important based on its dual role in breast cancer $[15,17]$. During early stages of cancer, TGF- $\beta$ may facilitate EMT processes while at later stages TGF- $\beta$ has an oncogenic role [15]. Thus, the EMT phenomenon may explain the metastatic dissemination of epithelial malignancies through the upregulation of "mesenchymal cadherins" [18]. Phorbol myristate acetate (PMA), an activator of protein kinase C, has been shown to induce EMT [19]. After traversing the bloodstream and entering the target metastatic site, the EMT cells could revert to an epithelial phenotype, known as the mesenchymal-to-epithelial transition (MET). The significance of EMT transition is that by preventing the process, metastasis may be diverted.

Targeted therapy against EMT processes has been investigated, and numerous compounds have been discovered that may inhibit transition toward a mesenchymal phenotype [20]. The use of a bioactive vimentin inhibitor, Withaferin-A, has been shown to induce apoptosis and inhibit tumor angiogenesis [16, 21]. Inhibitors of EMT include Klf4, which leads to increased E-cadherin expression and reduction in cellular invasion [22]. The Chinese herb salvianolic acid, which is used in renal disease, has been shown to inhibit EMT in the kidney through the modulation of TGF- $\beta$ signaling [20]. Silibinin, a flavanone from milk thistle extract, has been shown to downregulate ZEB1 and Slug in prostate cancer cells. It has also been shown to inhibit vimentin and matrix metalloproteinase 2 (MMP2) expression [23]. Phosphatidylinositol 3-kinase (PI3K)/Akt/mTOR signaling has also been shown to be a target for the inhibition of EMT processes [15]. Akt inhibitors, such as phosphatidylinositol 
ether lipid analogs, have been shown to promote MET in squamous cell carcinomas of the oral cavity [24]. Rapamycin has also been proposed to hinder EMT based on its inhibitory effects on mTOR [15]. These studies are still in their early stages, but the promising results warrant further research.

It is important to understand the normal structure of epithelial and mesenchymal tissues in order to appreciate the significance of EMT in cancer biology. Normal epithelium is well structured and typically overlies connective tissue constituting the basement membrane. These cells are normally held in tight interactions. Transformation of cells can lead to disruption of the epithelial architecture as malignant cells secrete matrix metalloproteinases to digest the underlying basement membrane, potentially inducing an inflammatory response reminiscent of wound healing [25]. The local inflammatory mediators and surrounding stroma can facilitate cancer progression [25].

\section{MSC-mediated oncoprotection}

Mesenchymal stem cell (MSC) interaction with the immune system is complex as MSCs have been shown to exert both stimulatory and inhibitory effects. The majority of MSCdependent immune responses are inhibitory, and this has been well established for many years [26]. The mechanisms of immune enhancement, however, have not been clearly identified until recently. Recent studies have demonstrated an enhancing effect by MSCs on immunomodulatory $\mathrm{T}$ cell subsets, specifically regulatory $\mathrm{T}$ cells $\left(\mathrm{T}_{\text {regs }}\right)$. These findings have been shown in various disease states. MSCs have been shown to facilitate the recruitment of $T_{\text {regs }}$ after approximately 2 weeks of co-culture [27]. The clinical implications of these findings have been recently demonstrated in graft tolerance studies, in which MSCs were shown to prevent rejection by increasing local $\mathrm{T}_{\text {regs }}$ [28]. MSCs have also been linked to increases in $\mathrm{T}_{\text {regs }}$ in colitis and rheumatoid arthritis, and they have been shown to act through $\mathrm{T}_{\text {regs }}$ to exert a protective effect in diabetes [29-31].

The role of MSCs in $\mathrm{T}_{\text {reg }}$ induction and expansion has major implications in cancer biology. MSCs have been shown to induce the production of $\mathrm{T}_{\text {regs }}$ when co-cultured with BCCs, allowing for immune subversion of BCCs in the bone marrow [32]. MSCs have been hypothesized to demonstrate an immune-protective effect in various cancers, known as oncoprotection, based on the idea that they can prevent the elimination of cancer cells by normal anti-tumor immune responses [32] (Fig. 2).

There is no current consensus on the mechanisms by which MSCs induce $T_{\text {regs }}$, but evidence has shown that TGF- $\beta$, a known polarizer of the $\mathrm{T}_{\text {reg }}$ phenotype, is partly responsible for the effects [32]. Since TGF- $\beta$ has a bimodal role in breast cancer progression, serving as a tumor suppressor at early stages and an oncogene at later stages, the role of MSC-derived TGF- $\beta$ is complex $[15,33,34]$. The secretion of TGF- $\beta$ from MSCs would be expected to protect against metastasis based on the inhibitory role of TGF- $\beta$ on epithelial cell cycle progression [33]. Expression of the HLA-G molecule, specifically soluble HLA-G5, from MSCs may also contribute to $\mathrm{T}_{\text {reg }}$ expansion in breast cancer [35]. MSCs can exert these effects on both autologous and allogeneic immune cells [31,32]. Based on these studies, cellular therapy involving MSCs is attractive for inflammatory and autoimmune states.

\section{Carcinoma-associated fibroblasts and the tumor microenvironment}

Concepts from the seed-and-soil hypothesis, proposed by Paget in the late nineteenth century, still stand true today as a possible explanation of microenvironmental factors that govern cancer growth [36]. Depending on the metastatic site, the breast cancer microenvironment can contain a variety of cell types, such as endothelial cells, macrophages, pericytes, lymphocytes, and stromal cells [37, 38]. Local invasion of cancer 
cells into resident tissue creates a nurturing environment for cancer cells in which growth and progression can occur relatively easily [39].

Perhaps, the most harmful players among cells of the tumor microenvironment are carcinoma-associated fibroblasts (CAFs), or myofibroblasts, which have been shown to facilitate cancer progression [40]. For example, in comparison to normal fibroblasts, CAFs have been shown to have greater stimulatory effects on triple-positive BCCs in threedimensional cultures [41]. CAFs and BCCs have a reciprocal relationship and are in constant interaction [41]. For example, the exposure of MSCs to conditioned media from $\mathrm{BCCs}$ can lead to MSC conversion into CAF-like cells expressing stromal cell-derived factor-1 (SDF-1), which has implications in BCC chemotaxis and metastasis to the bone marrow [37] (Fig. 3). These findings emphasize the importance of soluble factors in interactions between MSCs and BCCs and may explain the attraction that exists between these cell types $[32,37]$. Aside from their role in maintaining tumor survival, MSCs have been suspected to induce transformation in normal epithelial cells [42].

Interactions between MSC-derived CAFs and cancer cells are not limited to breast cancer. The interaction between MSCs and ovarian cancer cells has been studied, and evidence has shown that tumor-conditioned media from ovarian cancer cells result in enhanced expression of SDF- 1 and TGF- $\beta$ from MSCs and facilitate a conversion to a phenotype consistent with CAFs [38]. Synergy between the cancer cells and components of the tumor stroma may account for the difficulty in achieving therapeutic benefit [43].

Research into CAFs is still in its initial phases, but further studies may demonstrate that these cells are critical players in the survival and reactivation of dormant cancer cells in the bone marrow. Gene expression analyses of CAFs are underway and may hold the answers as to how these cells can be targeted at the molecular or genetic levels [39]. The markers for CAFs include, but are not limited to, $\alpha$-smooth muscle actin, fibroblast surface protein, and calponin [38]. These components are thought to have contractile function [37, 38]. However, the research is complicated by the heterogeneity in CAFs as CAFs have been shown to demonstrate variable responses to chemotherapy [40]. Microenvironmental factors may play a role in the chemosensitivity of cancer cells [40]. Microenvironment-mediated oncoprotection must be overcome before targeted therapy can emerge.

The origins of CAFs are uncertain, but a few ideas have been proposed. Some groups have suggested that these cells are distinct from normal resident fibroblast-based gene profiling studies $[25,39]$. The most apparent explanation is that CAFs may arise through direct oncogenic damage in normal fibroblasts. Other explanations hold that mesenchymal precursors may be recruited to the sites of malignancy and may facilitate cellular growth and tumor progression [44]. MSCs, for example, have been suggested as the precursor for CAFs and myofibroblasts that interact with BCCs [37]. MSCs gain expression of fibroblast surface protein and $\alpha$-smooth muscle actin after exposure to tumor-conditioned media [37]. HSCs have also been suggested to serve as the precursors for CAFs, but the data are unclear [45].

\section{Stem-like basis for human malignancies}

Throughout the past decade, there has been a tremendous upsurge of interest in the parallels between malignant cells and resident stem cells. Although the idea has been proposed as early as 50 years ago, these insights began to unravel only in 1997 with the discovery of a stem-like population in acute myelogenous leukemia [46]. These studies demonstrated that the malignant cells in leukemia possessed both phenotypic and functional properties of stem cells, such as the CD34+/CD38- phenotype, in addition to the functions of self-renewal, proliferation, and differentiation [46]. These findings concluded that oncogenic events may occur at early stages in the hematopoietic hierarchy. 
Since the discovery of these early findings, the field of oncology has recently shifted in favor of a hierarchal model for cancer, as compared to the traditional stochastic model, which held that oncogenes and tumor suppressors are disrupted by random mutations, deletions, additions, translocations, etc. that equally affect all cells within a tumor [47]. The hierarchal model, on the other hand, holds that particular cells within a lineage are susceptible to transformation, and these self-renewing cells give rise to heterogeneous progeny $[48,49]$. The cells at the pinnacle of the hierarchy can initiate de novo tumors and may account for resistance of cancer to chemotherapy and radiation [48, 49] (Fig. 4). Thus, one may define this model with the statement "not all cancer cells are created equally." Such hierarchal characteristics in hematological malignancies have suggested that solid tumors may also possess stem-like features, and this appears indeed to be the case. Given the upsurge of evidence regarding parallels between stem cells and cancer cells, the hierarchal model is currently well supported.

The genetic and molecular heterogeneity of cancer cells complicates the development of therapy and must be understood before treatments can reach the clinic [3]. Furthermore, shared intracellular signaling pathways and cell surface proteins between cancer cells and stem cells have also supported the idea of the existence of stem-like cells with tumorinitiating capacity [3]. For example, the expression of the neural and hematopoietic stem cells marker CD133 has been found in numerous transformed tissues, such as the colon, brain, and liver [50-52]. These markers may also demonstrate functionality as knockdown of CD133 in hepatocellular carcinoma has been shown to decrease production of matrix metalloproteinases [50]. As another example, dysregulation of the Hedgehog signaling pathway has been found in the cells of origin for basal cell carcinoma [53]. Indeed, microarray and genomic analyses have demonstrated striking similarities between stem cells and cancer cells in various tissue types [48]. A summary of the every piece of evidence for the existence of stem-like populations in cancer is out of the scope of this review, but evidence on the parallels between cancer cells and stem cells is rapidly accumulating.

A universal definition for the 'tumor stem cell' has not been established due to the high heterogeneity among tumors of various tissues and the lack of consensus regarding phenotype and function. However, it can be surmised that all of these cells should be able to harbor the properties of self-renewal and differentiation, which allows them to initiate heterogeneous tumors (Fig. 5). The self-renewing cells may represent a small proportion of the tumor, whereas the partially differentiated cells represent the bulk of the tumor [54]. These cells likely demonstrate dysregulation of proliferation and loss of normal cell cycle control [54, 55]. For example, stem-like populations in lung carcinomas have been found to demonstrate inactivation of $\mathrm{p} 16$ and pRb signaling, which are vital to G1 phase control [56]. Functionally, cancer stem cell populations are thought to demonstrate increased potential for invasion, migration, and metastasis [56, 57].

An understanding of the origin of stem-like populations in cancer may be important to the development of personalized therapy [58]. It is unclear whether these cells arise from reprograming of a partially differentiated cell type, allowing conversion to a high-grade phenotype, or whether normal resident stem cells undergo transformation [54, 59]. The concept is complicated by the idea that various stages of progenitors or transient-amplifying cells may also be the targets of transformation [54]. Knowledge of the origins of stem-like cells in cancers may highlight new therapeutic directions, such as differentiation therapy [54] (Table 2). Moreover, an understanding of these origins may provide insight into classification of cancer and may allow for better prognosis assessment [54]. Further complicating these ideas is that, within each tissue type, there may be particular cellular subtypes that give rise to stem-like tumor cells, such as the basal-like subgroup of breast cancer [5]. Basal-like breast cancers have been found to demonstrate aberrant activation of 
the canonical Wnt pathway, and Wnt signaling is a critical intracellular signal transduction pathway in stem cell biology [5].

Current investigations into the stem cell basis for malignancies seek to determine particular phenotypes that correspond with specific tissue types. Currently, there is no clear consensus on the stem cell markers that characterize different cancer subtypes [56]. The identification of such markers may facilitate prognostic assessment and may allow for the establishment of personalized and targeted therapy $[57,60]$. Tailored therapy thus far has included the use of oncolytic viruses, differentiation therapy, immuno-therapy, pharmacological disruption of intracellular signaling molecules, chemokine and ligand antagonism, epigenetic reprograming therapy, microenvironmental manipulation, and nanotechnology involving quantum dots for identification of stem-like populations [55, 61]. These therapeutic potentials are described in Table 2 . These treatments are geared at selectively destroying or inactivating malignant, self-renewing cells while sparing normal cells and healthy tissue [61]. The knowledge of the basic science behind these modalities of treatment is rapidly evolving, and these therapies may soon enter the clinic.

\section{References}

1. Baynes RD, Dansey RD, Klein JL, et al. High-dose chemotherapy and hematopoietic stem cell transplantation for breast cancer: past or future? Semin Oncol. 2001; 28:377-388. [PubMed: 11498831]

2. Weigelt B, Geyer FC, Reis-Filho JS. Histological types of breast cancer: how special are they? Mol Oncol. 2010; 4(3):192-208. [PubMed: 20452298]

3. Gatza ML, Lucas JE, Barry WT, et al. A pathway-based classification of human breast cancer. Proc Natl Acad Sci USA. 2010; 107:6994-6999. [PubMed: 20335537]

4. de Ronde J, Wessels L, Wesseling J. Molecular subtyping of breast cancer: ready to use? Lancet Oncol. 2010; 11:306-307. [PubMed: 20359657]

5. Khramtsov AI, Khramtsova GF, Tretiakova M, et al. Wnt/beta-catenin pathway activation is enriched in basal-like breast cancers and predicts poor outcome. Am J Pathol. 2010; 176(6):29112920. [PubMed: 20395444]

6. Riester M, Stephan-Otto Attolini C, Downey RJ, et al. A differentiation-based phylogeny of cancer subtypes. PLoS Comput Biol. 2010; 6(5):e1000777. [PubMed: 20463876]

7. Millikan RC, Newman B, Tse CK, et al. Epidemiology of basal-like breast cancer. Breast Cancer Res Treat. 2008; 109:123-139. [PubMed: 17578664]

8. Kim MJ, Ro JY, Ahn SH, et al. Clinicopathologic significance of the basal-like subtype of breast cancer: a comparison with hormone receptor and Her2/neu-overexpressing phenotypes. Hum Pathol. 2006; 37:1217-1226. [PubMed: 16938528]

9. Myal Y, Leygue E, Blanchard AA. Claudin 1 in breast tumorigenesis: revelation of a possible novel "claudin high" subset of breast cancers. J Biomed Biotechnol. 2010; 2010:956897. [PubMed: 20490282]

10. Tai P, Yu E, Joseph K. Prognostic significance of number of positive nodes: a long-term study of one to two nodes versus three nodes in breast cancer patients. Int J Radiat Oncol Biol Phys. 2010; 77:180-187. [PubMed: 20394852]

11. Chaffer CL, Thompson EW, Williams ED. Mesenchymal to epithelial transition in development and disease. Cells Tissues Organs. 2007; 185:7-19. [PubMed: 17587803]

12. Godde NJ, Galea RC, Elsum IA, et al. Cell polarity in motion: redefining mammary tissue organization through EMT and cell polarity transitions. J Mammary Gland Biol Neoplasia. 2010; 15(2):149-168. [PubMed: 20461450]

13. Takeyama Y, Sato M, Horio M, et al. Knockdown of ZEB1, a master epithelial-to-mesenchymal transition (EMT) gene, suppresses anchorage-independent cell growth of lung cancer cells. Cancer Lett. 2010; 296(2):216-224. [PubMed: 20452118] 
14. Dohadwala M, Wang G, Heinrich E, et al. The role of ZEB1 in the inflammation-induced promotion of EMT in HNSCC. Otolaryngol Head Neck Surg. 2010; 142:753-759. [PubMed: 20416468]

15. Taylor MA, Parvani JG, Schiemann WP. The pathophysiology of epithelial-mesenchymal transition induced by transforming growth factor-beta in normal and malignant mammary epithelial cells. J Mammary Gland Biol Neoplasia. 2010; 15(2):169-190. [PubMed: 20467795]

16. Lahat G, Zhu QS, Huang KL, et al. Vimentin is a novel anti-cancer therapeutic target; insights from in vitro and in vivo mice xenograft studies. PLoS One. 2010; 5(4):e10105. [PubMed: 20419128]

17. Ohashi S, Natsuizaka M, Wong GS, et al. Epidermal growth factor receptor and mutant p53 expand an esophageal cellular subpopulation capable of epithelial-to-mesenchymal transition through ZEB transcription factors. Cancer Res. 2010; 70:4174-4184. [PubMed: 20424117]

18. Berx G, van Roy F. Involvement of members of the cadherin superfamily in cancer. Cold Spring Harb Perspect Biol. 2009; 1(6):a003129. [PubMed: 20457567]

19. He H, Davidson AJ, Wu D, et al. Phorbol ester phorbol-12-myristate-13-acetate induces epithelial to mesenchymal transition in human prostate cancer ARCaP(E) cells. Prostate. 2010 (in press).

20. Wang QL, Tao YY, Yuan JL, et al. Salvianolic acid B prevents epithelial-to-mesenchymal transition through the TGF-beta1 signal transduction pathway in vivo and in vitro. BMC Cell Biol. 2010; 11:31. [PubMed: 20441599]

21. Bargagna-Mohan P, Hamza A, Kim YE, et al. The tumor inhibitor and antiangiogenic agent withaferin A targets the intermediate filament protein vimentin. Chem Biol. 2007; 4:623-634. [PubMed: 17584610]

22. Yori JL, Johnson E, Zhou G, et al. Kruppel-like factor 4 (KLF4) inhibits epithelial-tomesenchymal transition through regulation of E-cadherin gene expression. J Biol Chem. 2010; 285(22):16854-16863. [PubMed: 20356845]

23. Wu K, Zeng J, Li L, Fan J, Zhang D, Xue Y, Zhu G, Yang L, Wang X, He D. Silibinin reverses epithelial-to-mesenchymal transition in metastatic prostate cancer cells by targeting transcription factors. Oncol Rep. 2010; 23:1545-1552. [PubMed: 20428808]

24. Hong KO, Kim JH, Hong JS, et al. Inhibition of Akt activity induces the mesenchymal-toepithelial reverting transition with restoring E-cadherin expression in KB and KOSCC-25B oral squamous cell carcinoma cells. J Exp Clin Cancer Res. 2009; 28:28. [PubMed: 19243631]

25. Haviv I, Polyak K, Qiu W, et al. Origin of carcinoma associated fibroblasts. Cell Cycle. 2009; 8:589-595. [PubMed: 19182519]

26. Potian JA, Aviv H, Ponzio NM, et al. Veto-like activity of mesenchymal stem cells: functional discrimination between cellular responses to alloantigens and recall antigens. J Immunol. 2003; 171:3426-3434. [PubMed: 14500637]

27. Di Ianni M, Del Papa B, De Ioanni M, et al. Mesenchymal cells recruit and regulate T regulatory cells. Exp Hematol. 2008; 36:309-318. [PubMed: 18279718]

28. Casiraghi F, Azzollini N, Cassis P, et al. Pretransplant infusion of mesenchymal stem cells prolongs the survival of a semiallogeneic heart transplant through the generation of regulatory $\mathrm{T}$ cells. J Immunol. 2008; 181:3933-3946. [PubMed: 18768848]

29. Zhang Q, Shi S, Liu Y, et al. Mesenchymal stem cells derived from human gingiva are capable of immunomodulatory functions and ameliorate inflammation-related tissue destruction in experimental colitis. J Immunol. 2009; 183:7787-7798. [PubMed: 19923445]

30. Gonzalez MA, Gonzalez-Rey E, Rico L, et al. Treatment of experimental arthritis by inducing immune tolerance with human adipose-derived mesenchymal stem cells. Arthritis Rheum. 2009; 60:1006-1019. [PubMed: 19333946]

31. Madec AM, Mallone R, Afonso G, et al. Mesenchymal stem cells protect NOD mice from diabetes by inducing regulatory T cells. Diabetologia. 2009; 52:1391-1399. [PubMed: 19421731]

32. Patel SA, Meyer JR, Greco SJ, et al. Mesenchymal stem cells protect breast cancer cells through regulatory T cells: role of mesenchymal stem cell-derived TGF-beta. J Immunol. 2010; 184(10): 5885-5894. [PubMed: 20382885] 
33. Yang YA, Dukhanina O, Tang B, et al. Lifetime exposure to a soluble TGF-beta antagonist protects mice against metastasis without adverse side effects. J Clin Invest. 2002; 109:1607-1615. [PubMed: 12070308]

34. Tang B, Vu M, Booker T, et al. TGF-beta switches from tumor suppressor to prometastatic factor in a model of breast cancer progression. J Clin Invest. 2003; 112:112-1116.

35. Selmani Z, Naji A, Zidi I, et al. Human leukocyte antigen-G5 secretion by human mesenchymal stem cells is required to suppress $T$ lymphocyte and natural killer function and to induce CD4+CD25highFOXP3+ regulatory T cells. Stem Cells. 2008; 26:212-222. [PubMed: 17932417]

36. Paget S. The distributions of secondary growths in cancer of the breast. Lancet. 1889; 1:571-573.

37. Mishra PJ, Mishra PJ, Humeniuk R, et al. Carcinoma-associated fibroblast-like differentiation of human mesenchymal stem cells. Cancer Res. 2008; 68:4331-4339. [PubMed: 18519693]

38. Jeon ES, Moon HJ, Lee MJ, et al. Cancer-derived lyso-phosphatidic acid stimulates differentiation of human mesenchymal stem cells to myofibroblast-like cells. Stem Cells. 2008; 26:789-797. [PubMed: 18065393]

39. Bauer M, Su G, Casper C, et al. Heterogeneity of gene expression in stromal fibroblasts of human breast carcinomas and normal breast. Oncogene. 2010; 29:1732-1740. [PubMed: 20062080]

40. Sonnenberg M, van der Kuip H, Haubeis S, et al. Highly variable response to cytotoxic chemotherapy in carcinoma-associated fibroblasts (CAFs) from lung and breast. BMC Cancer. 2008; 8:364. [PubMed: 19077243]

41. Su G, Blaine SA, Qiao D, et al. Membrane type 1 matrix metalloproteinase-mediated stromal syndecan-1 shedding stimulates breast carcinoma cell proliferation. Cancer Res. 2008; 68:95589565. [PubMed: 19010933]

42. Olumi AF, Grossfeld GD, Hayward SW, et al. Carcinoma-associated fibroblasts direct tumor progression of initiated human prostatic epithelium. Cancer Res. 1999; 59:5002-5011. [PubMed: 10519415]

43. Karnoub AE, Dash AB, Vo AP, et al. Mesenchymal stem cells within tumour stroma promote breast cancer metastasis. Nature. 2007; 449:557-563. [PubMed: 17914389]

44. Lama VN, Phan SH. The extrapulmonary origin of fibroblasts: stem/progenitor cells and beyond. Proc Am Thorac Soc. 2006; 3:373-376. [PubMed: 16738203]

45. LaRue AC, Masuya M, Ebihara Y, et al. Hematopoietic origins of fibroblasts: I. In vivo studies of fibroblasts associated with solid tumors. Exp Hematol. 2006; 34:208-218. [PubMed: 16459189]

46. Bonnet D, Dick JE. Human acute myeloid leukemia is organized as a hierarchy that originates from a primitive hematopoietic cell. Nat Med. 1997; 3:730-737. [PubMed: 9212098]

47. Reya T, Morrison SJ, Clarke MF, et al. Stem cells, cancer, and cancer stem cells. Nature. 2001; 414:105-111. [PubMed: 11689955]

48. Marquardt JU, Thorgeirsson SS. Stem cells in hepatocarcinogenesis: evidence from genomic data. Semin Liver Dis. 2010; 30:26-34. [PubMed: 20175031]

49. Hubbard S, Gargett C. A cancer stem cell origin for human endometrial carcinoma? Reproduction. 2010; 140(1):23-32. [PubMed: 20089663]

50. Kohga K, Tatsumi T, Takehara T, et al. Expression of CD133 confers malignant potential by regulating metalloproteinases in human hepatocellular carcinoma. J Hepatol. 2010; 52(6):872-879. [PubMed: 20395004]

51. Yasuda H, Tanaka K, Saigusa S, et al. Elevated CD133, but not VEGF or EGFR, as a predictive marker of distant recurrence after preoperative chemoradiotherapy in rectal cancer. Oncol Rep. 2009; 22:709-717. [PubMed: 19724847]

52. Brown CE, Starr R, Martinez C, et al. Recognition and killing of brain tumor stem-like initiating cells by CD8+ cytolytic T cells. Cancer Res. 2009; 69:8886-8893. [PubMed: 19903840]

53. Youssef KK, Van Keymeulen A, Lapouge G, et al. Identification of the cell lineage at the origin of basal cell carcinoma. Nat Cell Biol. 2010; 12:299-305. [PubMed: 20154679]

54. Groner B, Vafaizadeh V, Brill B, et al. Stem cells of the breast and cancer therapy. Womens Health. 2010; 6:205-219.

55. Giuffrida D, Rogers I. Targeting cancer stem cell lines as a new treatment of human cancer. Recent Pat Anticancer Drug Discov. 2010; 5(3):205-218. [PubMed: 20402651] 
56. Arifin M, Tanimoto K, Putra AC, et al. Carcinogenesis and cellular immortalization without persistent inactivation of p16/Rb pathway in lung cancer. Int J Oncol. 2010; 36:1217-1227. [PubMed: 20372796]

57. Ahmed N, Abubaker K, Findlay J, et al. Epithelial mesenchymal transition and cancer stem celllike phenotypes facilitate chemoresistance in recurrent ovarian cancer. Curr Cancer Drug Targets. 2010; 10:268-278. [PubMed: 20370691]

58. Luo J, Yin X, Ma T, et al. Stem cells in normal mammary gland and breast cancer. Am J Med Sci. 2010; 339:366-370. [PubMed: 20224316]

59. Castellanos A, Vicente-Dueñas C, Campos-Sánchez E, et al. Cancer as a reprogramming-like disease: Implications in tumor development and treatment. Semin Cancer Biol. 2010; 20(2):93-97. [PubMed: 20188174]

60. Shabason JE, Camphausen K. Cancer stem cells as a prognostic indicator for glioblastoma multiforme. Biomark Med. 2010; 4:127-128. [PubMed: 20387308]

61. ten Cate B, de Bruyn M, Wei Y, et al. Targeted elimination of leukemia stem cells; a new therapeutic approach in hemato-oncology. Curr Drug Targets. 2010; 11:95-110. [PubMed: 20017722]

62. Carey LA, Perou CM, Livasy CA, et al. Race, breast cancer subtypes, and survival in the Carolina Breast Cancer Study. JAMA. 2006; 295:2492-2502. [PubMed: 16757721]

63. Eriksson M, Guse K, Bauerschmitz G, et al. Oncolytic adenoviruses kill breast cancer initiating CD44+CD24-/low cells. Mol Ther. 2007; 15:2088-2093. [PubMed: 17848962]

64. Redova M, Chlapek P, Loja T, et al. Influence of LOX/COX inhibitors on cell differentiation induced by all-trans retinoic acid in neuroblastoma cell lines. Int J Mol Med. 2010; 25:271-280. [PubMed: 20043138]

65. Yamashita T, Honda M, Nio K, et al. Oncostatin m renders epithelial cell adhesion moleculepositive liver cancer stem cells sensitive to 5-Fluorouracil by inducing hepatocytic differentiation. Cancer Res. 2010; 70(11):4687-4697. [PubMed: 20484035]

66. Salnikov AV, Groth A, Apel A, et al. Targeting of cancer stem cell marker EpCAM by bispecific antibody EpCAMxCD3 inhibits pancreatic carcinoma. J Cell Mol Med. 2009; 13:4023-4033. [PubMed: 20196789]

67. Tazzari PL, Tabellini G, Ricci F, et al. Synergistic proapoptotic activity of recombinant TRAIL plus the Akt inhibitor Perifosine in acute myelogenous leukemia cells. Cancer Res. 2008; 68:9394-9403. [PubMed: 19010914]

68. Zhang M, Atkinson RL, Rosen JM. Selective targeting of radiation-resistant tumor-initiating cells. Proc Natl Acad Sci USA. 2010; 107:3522-3527. [PubMed: 20133717]

69. Grymula K, Tarnowski M, Wysoczynski M, et al. Overlapping and distinct role of CXCR7-SDF-1/ ITAC and CXCR4-SDF-1 axes in regulating metastatic behavior of human rhabdo-myosarcomas. Int J Cancer. 2010; 127(11):2554-2568. [PubMed: 20162608]

70. Dillmann F, Veldwijk MR, Laufs S, et al. Plerixafor inhibits chemotaxis toward SDF-1 and CXCR4-mediated stroma contact in a dose-dependent manner resulting in increased susceptibility of BCR-ABL+ cell to Imatinib and Nilotinib. Leuk Lymphoma. 2009; 50:1676-1686. [PubMed: 19657955]

71. Galanzha EI, Kim JW, Zharov VP. Nanotechnology-based molecular photoacoustic and photothermal flow cytometry platform for in vivo detection and killing of circulating cancer stem cells. J Biophotonics. 2009; 2:725-735. [PubMed: 19957272] 
Epithelial-to-mesenchymal transition

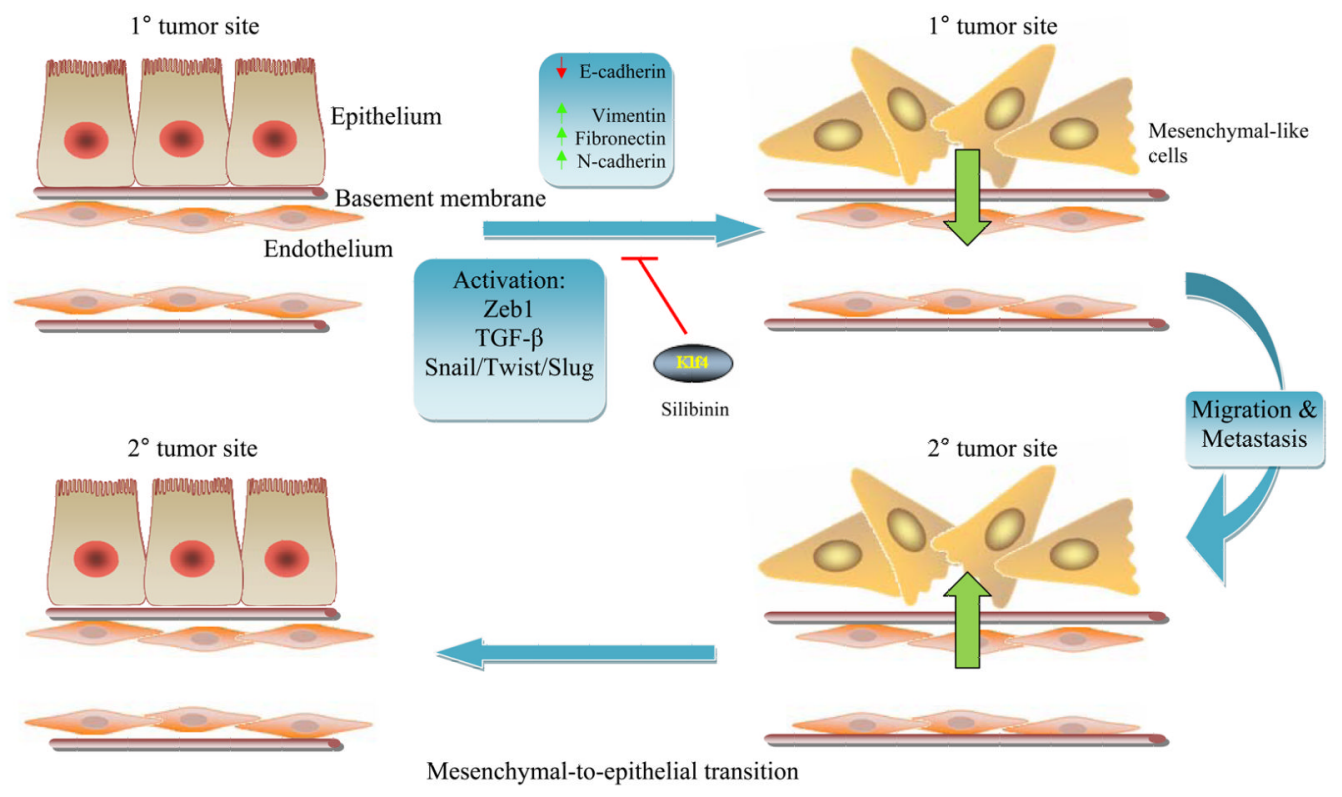

Fig. 1.

Polarized epithelial cells at the primary tumor site may acquire mesenchymal-like properties enabling these cells to transverse the basement membrane and enter the vasculature $[14,15$, 17]. Once these cells migrate to distant metastatic sites and pass into the interstitial space, mesenchymal-to-epithelial reversion may occur, facilitating establishment of these malignant cells permanently in the secondary site where they can establish dormancy [14, $15,17]$ 


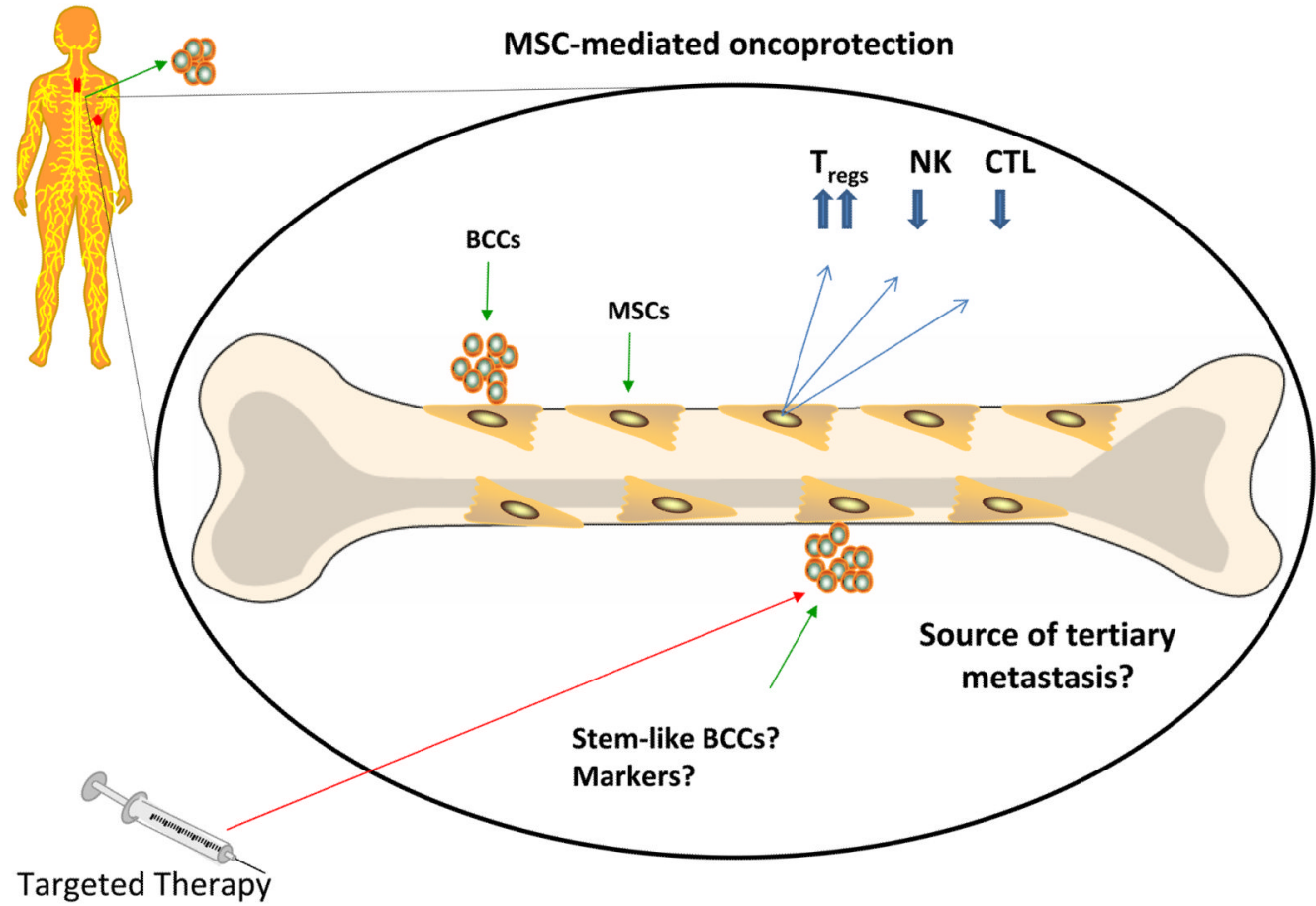

Fig. 2.

Mesenchymal stem cells within the bone marrow stroma exert an oncoprotective role on migrant breast cancer cells through complex interactions with components of the immune system. It is possible that a small subpopulation of breast cancer cells responsible for metastasis harbors stem-like qualities, and this subpopulation may serve as a target for therapy [29-32]. $T_{\text {regs }}$ regulatory T cells, $N K$ natural killer cells, $C T L$ cytotoxic T lymphocytes 


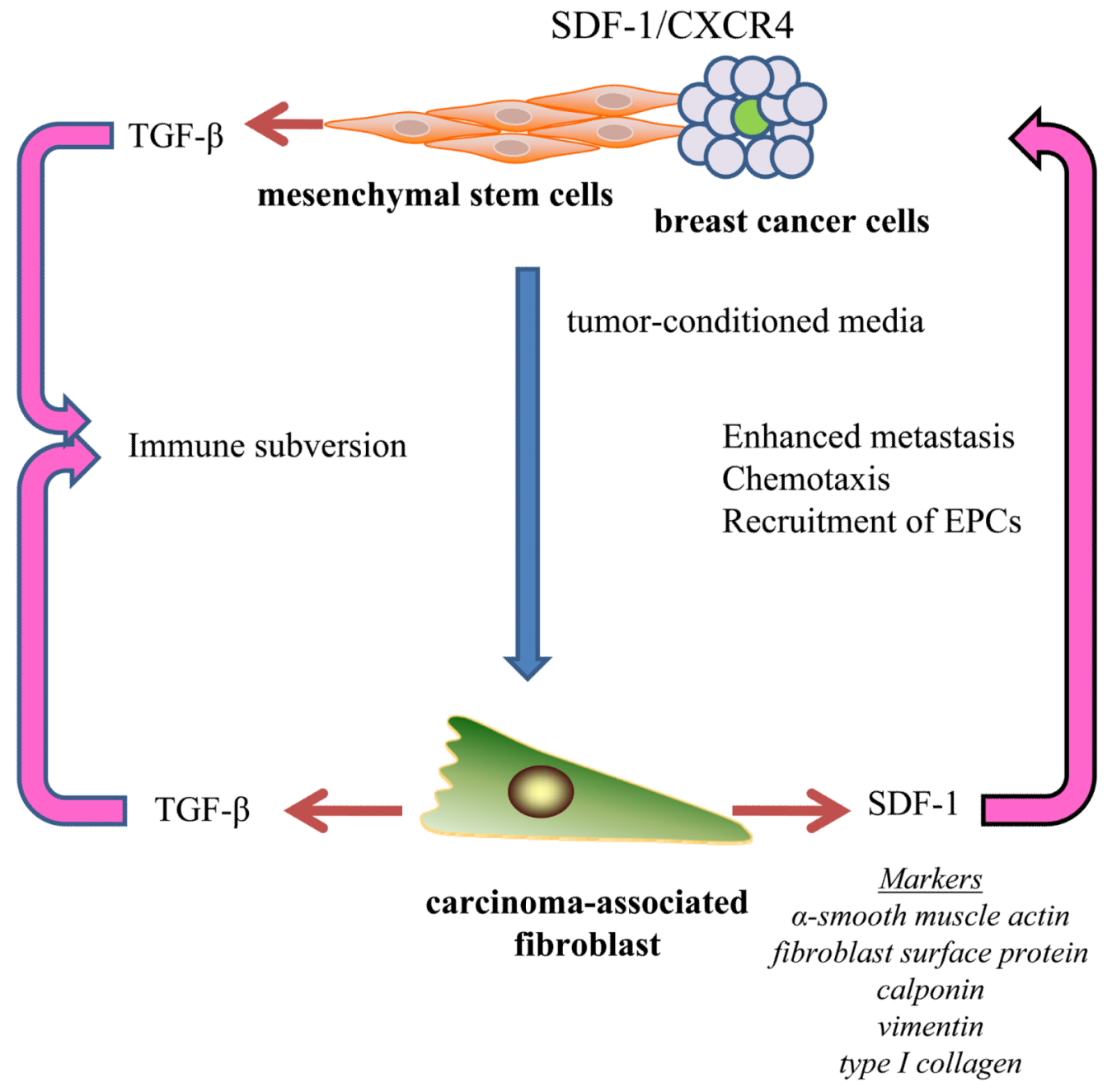

Fig. 3.

The generation of carcinoma-associated fibroblasts from mesenchymal stem cells provides a protective and trophic environment for breast cancer progression. Autocrine signaling loops involving TGF- $\beta$ and SDF-1 promote immune suppression and chemotaxis, respectively, which have important implications in breast cancer dormancy in metastatic sites. The targeting of these signaling loops may serve as an avenue through which breast cancer remission or treatment can be achieved 


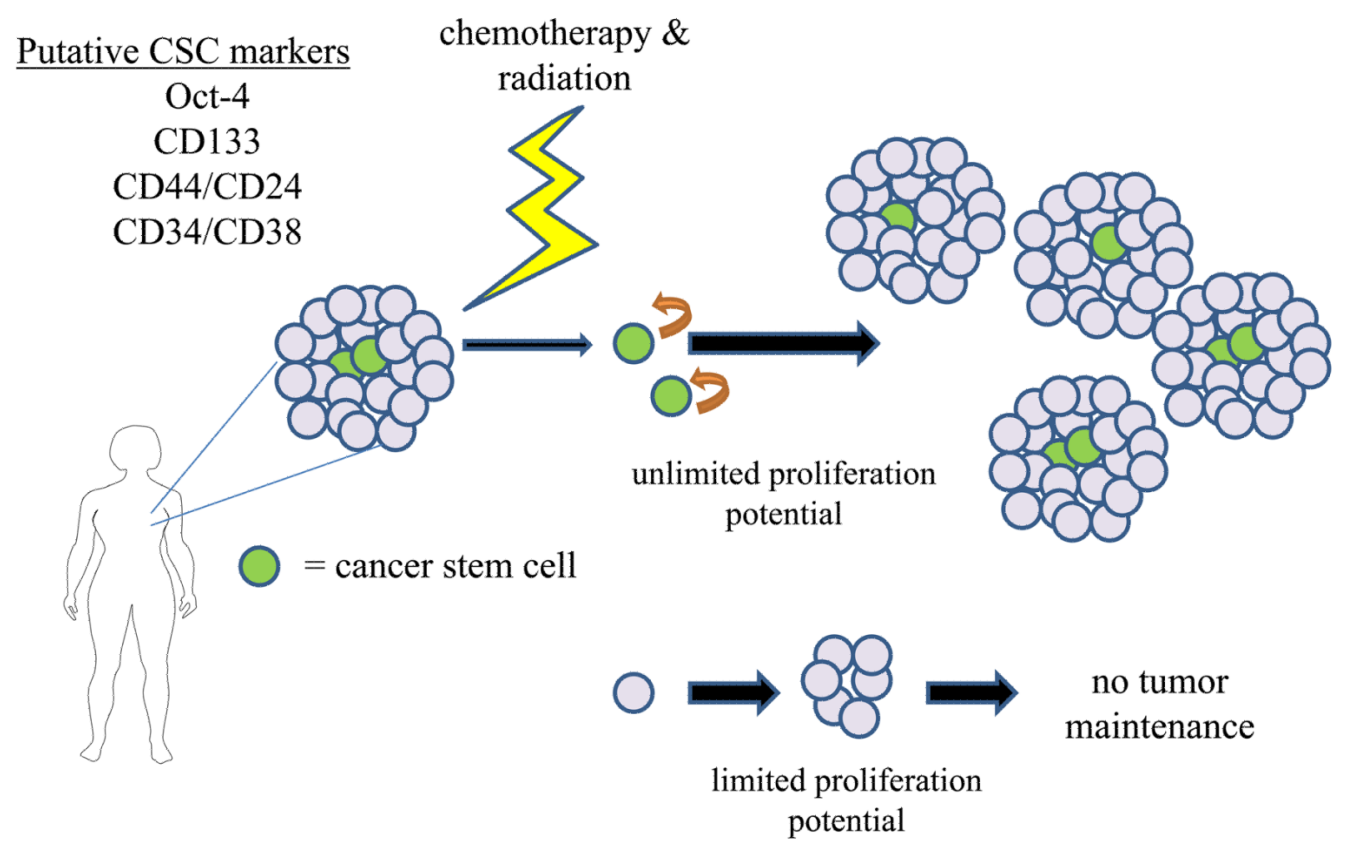

Fig. 4.

Current chemotherapeutic and radiation therapies target the rapidly dividing cells which make up the bulk of the tumor, but may leave behind the slower cycling stem-like cells. These cells are expected to self-renew and give rise to new tumors limitlessly [48, 49]. The pluripotency marker Oct-4 is shown here as a potential marker of these cancer-initiating cells 
A.

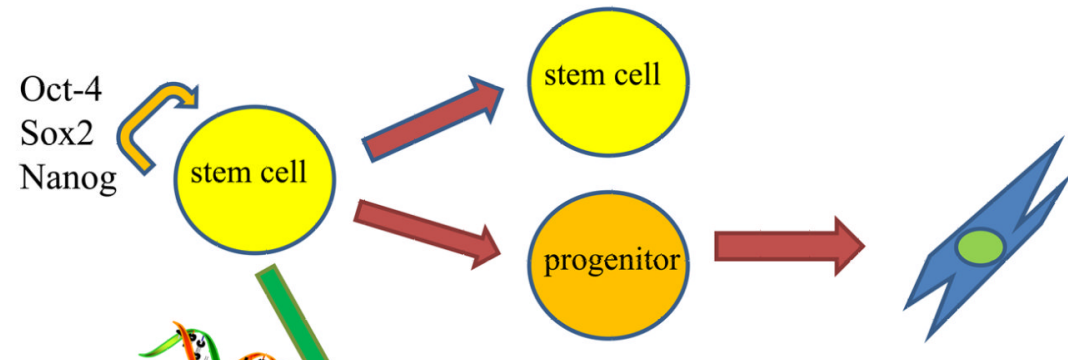

Genomic damage?

B.

Oncogenesis

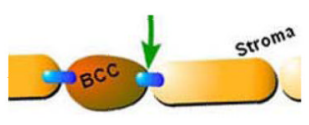

Gap junctions with stroma?
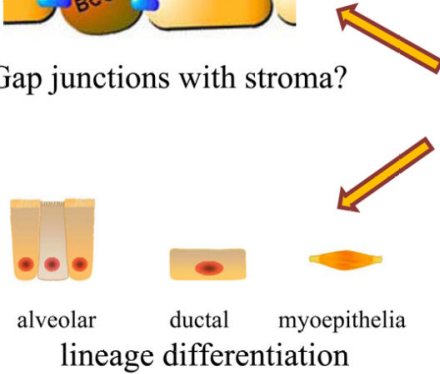

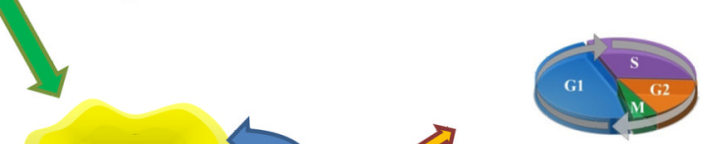

Cell cycle quiescence?

Self-renewing breast cancer cell

differentiated cell

Fig. 5.

a Resident stem cells of a given tissue have the ability to undergo asymmetric division in which the stem cell self-renews and gives rise to a committed progenitor. Upon loss of genomic stability, resident stem cells may become transformed. b Stem-like breast cancer cells, which undergo self-renewal and multi-lineage differentiation, may have similar properties to pluripotent stem cells and can give rise to serial tumorspheres $[54,55]$. They are thought to establish gap junctions with bone marrow stroma and adopt a quiescent phenotype, serving as a source of tertiary metastasis 


\section{Table 1}

Heterogeneity in breast cancer as evident from classification scheme

\begin{tabular}{ll}
\hline Major classification & Phenotype \\
\hline Luminal A & ER+/PR+, HER2- \\
Luminal B & ER+/PR+, HER2+ \\
Basal-like & ER-/PR-, HER2-, Ck5/6+, HER1+ \\
HER2+ & ER-/PR-, HER2+ \\
Unclassified & ER-/PR-, HER2-, Ck5/6-, HER1- \\
\hline
\end{tabular}

Reference: [62]

$H E R 2$ human epidermal growth factor receptor 2, $E R$ estrogen receptor, $P R$ progesterone receptor, $C k$ cytokeratin 
Table 2

Potential therapeutic avenues against stem-like cancer cells

\begin{tabular}{ll}
\hline Therapeutic modality & Tissue-specific example \\
\hline $\begin{array}{l}\text { Oncolytic viral therapy } \\
\text { Differentiation therapy }\end{array}$ & $\begin{array}{l}\text { Capsid-modified adenoviral vectors against CD44+/CD24- breast cancer cells } \\
\text { All-trans retinoic acid treatment for neuroblastoma; Oncostatin M (IL-6-related) for } \\
\text { hepatocellular carcinoma }\end{array}$ \\
[64, 65] & $\begin{array}{l}\text { Monoclonal antibodies against tumors for neutralization, inhibition, or cytotoxicity } \\
\text { purposes; EpCAM antibody for stem-like population in pancreatic cancer }\end{array}$ \\
[66] & $\begin{array}{l}\text { Perifosine for Akt inhibition in leukemic stem cells and radioresistant breast cancer } \\
\text { cells }\end{array}$ \\
$\begin{array}{l}\text { [67, 68] } \\
\text { intracellular signaling }\end{array}$ & $\begin{array}{l}\text { Disruption of bone marrow-mediated oncoprotection by inhibiting CXCR4/SDF-1 } \\
\text { signaling in rhabdomyosarcoma and leukemia }\end{array}$ \\
Microenvironmental manipulation & $\begin{array}{l}\text { Highly sensitive detection of disseminated breast tumor cells of CD44+ phenotype } \\
\text { using photoacoustic flow cytometry }\end{array}$ \\
[71]
\end{tabular}

